\title{
Analysis of Optical Waveguides with High-Reflection Coatings Using the FD-TD Method
}

\author{
Junji Yamauchi, Member, IEEE, Hiroyuki Kanbara, and Hisamatsu Nakano, Fellow, IEEE
}

\begin{abstract}
An optical waveguide with a high-reflection coating is investigated using the finite-difference time-domain (FD-TD) method. The obtained reflectivities are compared with those from the transmission matrix method. Calculation shows the existence of the field propagating along the coating layers, resulting in deterioration of reflectivity, particularly for TM wave.
\end{abstract} ing.

Index Terms - Finite-difference method, high-reflection coat-

\section{INTRODUCTION}

A HIGH-REFLECTION (HR) mirror consisting of alternating dielectric layers [1]-[3] is useful in many applications, since mirrors made of metallic films often cause severe heating problems. So far, the transmission matrix method (TMM) [4] has widely been used to design an HR coating. However, the TMM assumes a homogeneous medium in the transverse direction, so that the estimated results do not necessarily agree with the experimental results [1], particularly for a waveguide facet with an HR coating. Furthermore, the TMM does not give information on polarization dependence at normal incidence.

In this letter, we numerically investigate the reflectivity of an HR coating consisting of quarter-wavelength $\left(\lambda_{g} / 4\right)$ layers of two different materials. For the analysis, the finite-difference time-domain (FD-TD) method [5]-[7] is used, since it allows us to study the reflection of optical power from alternating layered media [8]. It is found that the reflectivity for the TM wave considerably differs from those obtained from the TMM. Further calculation shows the existence of the field propagating along the coating layers, and this results in deterioration of reflectivity, particularly for the TM wave.

\section{Configuration And Numerical Method}

For simplicity, we consider a two-dimensional waveguide whose facet is coated with alternating dielectric layers, as shown in Fig. 1. We choose $\mathrm{Al}_{2} \mathrm{O}_{3}$ as the low-index dielectric adjacent to the facet and $\mathrm{Si}$ as the high-index dielectric material, i.e., $n_{L}=1.7$ and $n_{H}=3.5$. The refractive indices of the core and the cladding are taken to be $n_{\mathrm{CO}}=3.6$ and $n_{\mathrm{CL}}=3.24$, respectively.

Manuscript received May 27, 1997; revised June 30, 1997.

The authors are with the College of Engineering, Hosei University, 3-7-2, Kajino-cho, Koganei, Tokyo 184, Japan.

Publisher Item Identifier S 1041-1135(98)00438-8.

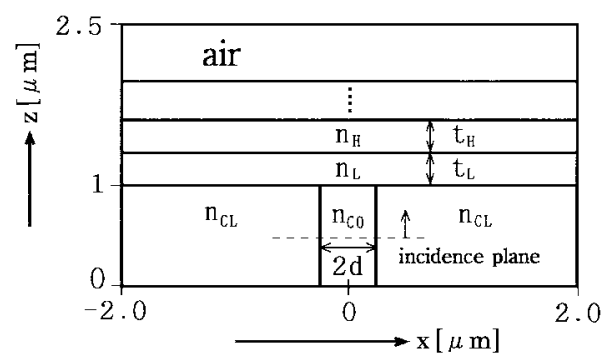

Fig. 1. Configuration of an HR-coated waveguide.

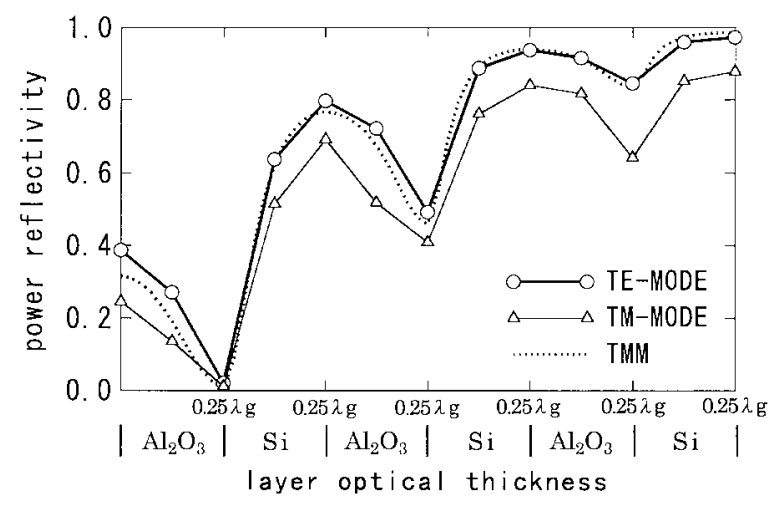

Fig. 2. Power reflectivity at $\lambda=0.8 \mu \mathrm{m}$ as a function of layer thickness for alternating layers.

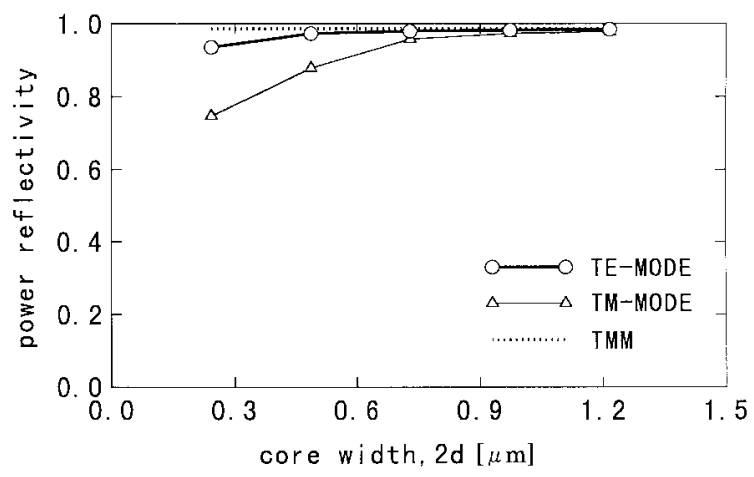

Fig. 3. Power reflectivity as a function of core width.

The FD-TD method is used to analyze the field distribution in this structure. The incident field $\left(\mathrm{TE}_{0}\right.$ or $\mathrm{TM}_{0}$ mode) is excited toward the $+z$ direction from the incidence plane indicated by the dotted line in Fig. 1, so that the computation 

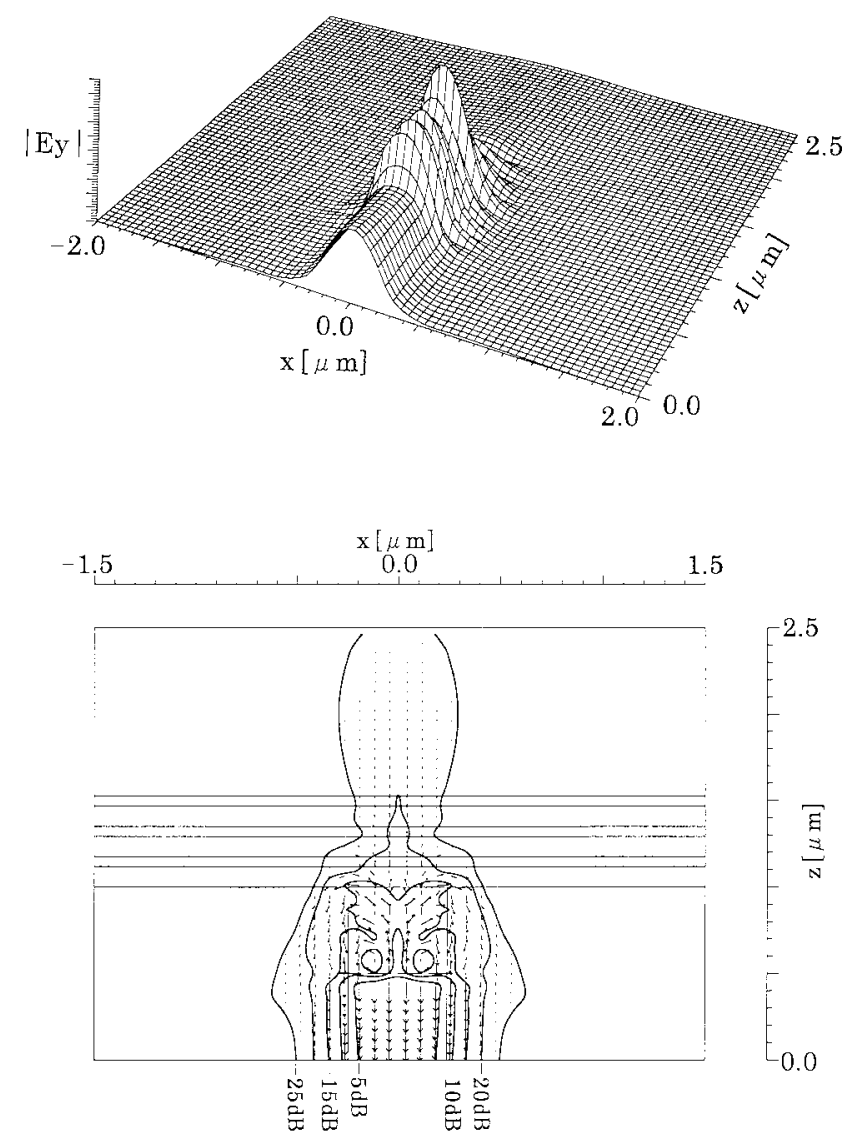

(a)
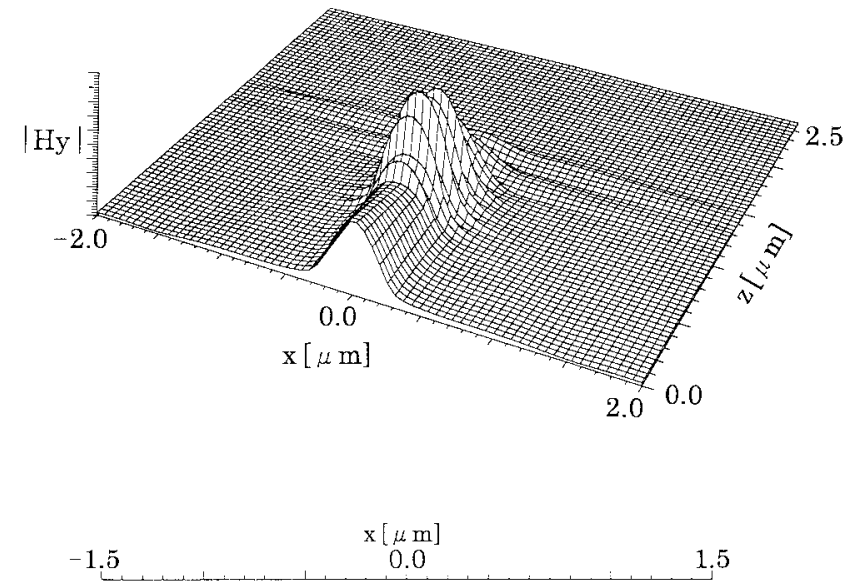

1.5

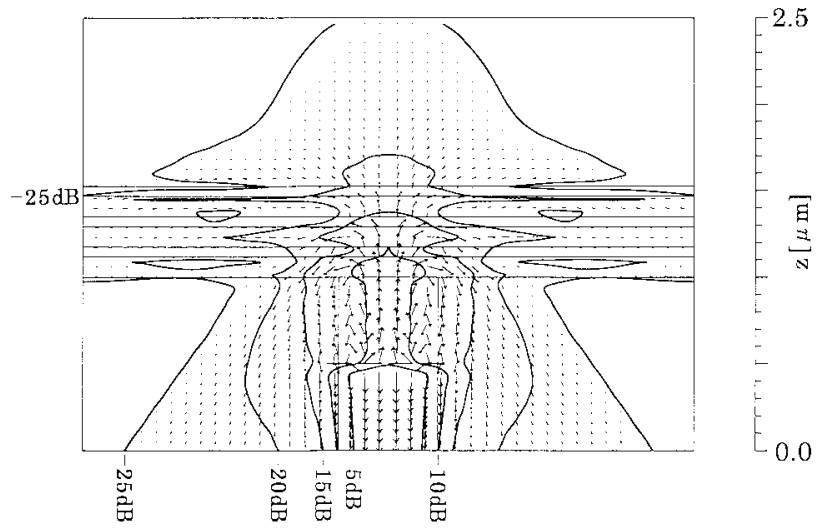

(b)

Fig. 4. Steady-state field distributions and corresponding Poynting vector plots ( $c t=15 \mu \mathrm{m}$ ). (a) $\mathrm{TE}_{0}$ mode and (b) $\mathrm{TM}_{0}$ mode.

region is divided into reflected- and total-field regions [6]. The computational parameters are as follows: $\Delta x \simeq 0.012 \mu \mathrm{m}$, $\Delta z=0.004 \mu \mathrm{m}$, and $c \Delta t=0.001 \mu \mathrm{m}$, where $c$ is the velocity of light in free space. To avoid undesirable reflection from the computational boundaries, the perfectly matched layer (PML) [9] is placed at the edge of the computational region. The effectiveness of the PML has already been verified in optical waveguiding problems [7], [10]. In this analysis, the reflection coefficient is taken to be $R(0)=10^{-8}$, and the number of PML layers is 16 .

\section{RESULTS}

We first consider reflectivities as a function of layer thickness for alternating layers for $\mathrm{Al}_{2} \mathrm{O}_{3}\left(\lambda_{g} / 4\right.$ thick $)$ and $\mathrm{Si}\left(\lambda_{g} / 4\right.$ thick) sandwich. The core width of the waveguide is taken as $2 d \simeq 0.487 \mu \mathrm{m}$, which corresponds to a normalized frequency of $V=3.0$ at $\lambda=0.8 \mu \mathrm{m}$. Fig. 2 shows the reflectivities of the fundamental modes for TE and TM waves at $\lambda=0.8$ $\mu \mathrm{m}$. For comparison, the data obtained from the TMM is also presented. From Fig. 2 we can see how the proper combination of $\lambda_{g} / 4$ layers leads to a reasonable reflectivity. The results obtained from the TE wave are close to those obtained from the TMM. It should be noted, however, that the results for the TM wave differ from the TMM results. For example, the maximum reflectivities observed at six layers are 0.97 for the TE wave and 0.88 for the TM wave, while the result from the

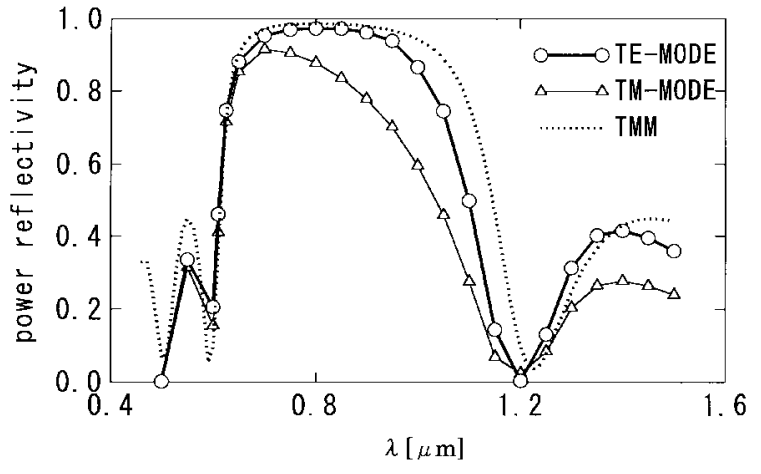

Fig. 5. Power reflectivity as a function of wavelength $\lambda$.

TMM is 0.99. Incidentally, it is worth mentioning that a single layer coating by $\mathrm{Al}_{2} \mathrm{O}_{3}$ acts as an antireflection coating [11], whose reflectivity is less than 0.02 .

The field profiles for both polarizations spread, as the core width is increased. Note that the TMM assumes a plane wave in a homogeneous medium. Therefore, it is expected that the results for both polarizations obtained from the FD-TD method approach the value estimated from the TMM with increase in the core width. Fig. 3 shows the power reflectivity as a function of core width. The results are for six layers when the fundamental mode is excited. The core width ranges from $2 d \simeq 0.243 \mu \mathrm{m}$ to $1.217 \mu \mathrm{m}$, corresponding to a normalized frequency range from $V=1.5$ to 7.5 . As expected, the 
calculated results for both polarizations approach the TMM result with increase in the core width. When the core width is larger than $2 d=1 \mu \mathrm{m}(V=6)$, the difference among them is almost negligible.

Typical steady-state field distributions for both polarizations are shown in Fig. 4. The results are for a six-layer stack with $2 d \simeq 0.487 \mu \mathrm{m}$. Corresponding Poynting vector plots are also presented. The arrow length is scaled according to the power magnitude, in which the power of less than $-25 \mathrm{~dB}$ is omitted. It is found that some power propagates along the coating layers. Comparison between Fig. 4(a) and (b) shows that the power coupled into the coating layers is more pronounced in the TM wave. It should be noted that the alternating dielectric layers on a waveguide facet can be regarded as a leaky wave structure [12], so that the field propagating along the coating layers tends to decay as it propagates. Furthermore, the reflected field from the coating involves radiation modes, particularly for the TM wave. The low reflectivity in the TM wave can be explained by these observations.

Final consideration is given to the wavelength dependence of reflectivity. Fig. 5 shows the results for a six-layer stack with $2 d \simeq 0.487 \mu \mathrm{m}$. Each coating thickness is fixed to be a quarter-wavelength at $\lambda=0.8 \mu \mathrm{m}$. The TE wave exhibits high reflectivities over a wide spectral range from 0.7 to 1.0 $\mu \mathrm{m}$, showing good agreement with the TMM results. It should be noted, however, that at wavelengths of larger than $1.0 \mu \mathrm{m}$, agreement is only qualitative. The tendency of our numerical results is similar to the experimental observation reported previously [1] (unfortunately with no information about the waveguide dimensions). Fig. 5 also shows that the data for the TM wave considerably deviates from the TMM result.

\section{CONCLUSION}

The power reflectivity from an HR-coated waveguide consisting of alternating dielectric layers has been investigated using the finite-difference time-domain method. The numerical results are compared with those obtained from the conventional TMM. It is found that the field propagating along the coating layers reduces reflectivity, particularly for TM polarization. The TMM gives reliable results, only when the core width of the waveguide is large enough, e.g., $V \geq 6$.

\section{REFERENCES}

[1] M. Ettenberg, "A new dielectric facet reflector for semiconductor lasers," Appl. Phys. Lett., vol. 32, no. 11, pp. 724-725, 1978.

[2] T. Ohtoshi, T. Kawano, Y. Sasaki, T. Kajimura, N. Chinone, and M. Nakamura, "High-power visible GaAlAs lasers with self-aligned strip buried heterostructure," J. Appl. Phys., vol. 56, no. 9, pp. 2491-2496, 1984.

[3] K. Hamada, M. Wada, H. Shimizu, M. Kume, F. Susa, T. Shibutani, N. Yoshikawa, K. Itoh, G. Kano, and I. Teramoto, "A 0.2 w CW laser with buried twin-ridge substrate structure," IEEE J. Quantum Electron., vol. QE-21, pp. 623-627, 1985.

[4] P. Yeh, Optical Waves in Layered Media. New York: Wiley, 1988, ch.

[5] K. S. Yee, "Numerical solution of initial boundary value problems involving Maxwell's equations in isotropic media," IEEE Trans. Antennas Propagat., vol. AP-14, pp. 302-307, 1966.

[6] S. T. Chu, W. P. Huang, and S. K. Chaudhuri, "Simulation and analysis of waveguide based optical integrated circuits," Comp. Phys. Commun., vol. 68, pp. 451-484, 1991.

[7] A. Taflove, Computational Electrodynamics: The Finite-Difference Time-Domain Method. Norwood, MA: Artech House, 1995.

[8] S. C. Hagness, R. M. Joseph, and A. Taflove, "Subpicosecond electrodynamics of distributed Bragg reflector microlasers: Results from finite-difference time-domain simulations," Radio Sci., vol. 31, pp. 931-941, 1996.

[9] J. P. Berenger, "A perfectly matched layer for the absorption of electromagnetic waves," J. Comput. Phys., vol. 114, pp. 185-200, 1994.

[10] C. E. Reuter, R. M. Joseph, E. T. Thiele, D. S. Katz, and A. Taflove, "Ultrawideband absorbing boundary condition for termination of waveguiding structures in FDTD simulations," IEEE Microwave Guided Wave Lett., vol. 4, pp. 344-346, 1994.

[11] J. Yamauchi, M. Mita, S. Aoki, and H. Nakano, "Analysis of antireflection coatings using the FD-TD method with the PML absorbing boundary condition," IEEE Photon. Technol. Lett., vol. 8, pp. 239-241, 1996.

[12] M. Miyagi, Introduction to Optical Fiber Transmission. Tokyo, Japan: Shokodo, 1991. 